\title{
Multi-enzymes Production Studies in Single Tray Solid State Fermentation with Opened and Closed System
}

\author{
Musaalbakri Abdul Manan ${ }^{1,2}$ and Colin $\mathrm{Webb}^{2}$ \\ 1. Biotechnology and Nanotechnology Research Centre, Malaysian Agricultural Research and Development Institute (MARDI), \\ Persiaran MARDI-UPM, 43400 Serdang, Selangor, Malaysia \\ 2. School of Chemical Engineering and Analytical Science, The University of Manchester, Oxford Road, Manchester M13 9PL, \\ United Kingdom
}

\begin{abstract}
The robustness of A. awamori and A. oryzae as enzyme producers is exploited in fungal fermentation on agricultural solid waste. High-level production of extracellular glucoamylase, protease, cellulase and xylanase has been achieved. Three different types of 'waste' solids (wheat bran, soybean hulls and rapeseed meal) have been used in studies of solid state fermentation (SSF). The enzymes could be produced in significant levels by continuously supplying oxygen $\left(\mathrm{O}_{2}\right)$ through the tray system known as "closed" and "opened" tray systems. A perforated tray system was developed in this study that permits direct access to $\mathrm{O}_{2}$. Testing the tray system with different perforated mesh aperture sizes in this study did not yield different results in growth performance of A. awamori and A. oryzae. A. awamori and A. oryzae can be very versatile in producing various enzymes with different substrates with different starch, protein, hemicellulose and cellulose contents. These studies indicate that A. awamori is more suitable for the efficient production of multiple enzymes in the closed system including xylanase and cellulase, while the production of glucoamylase and protease is superior in the opened system. A. oryzae is more suitable for the efficient production of protease and cellulase in the closed system, while the production of protease is more favourable the opened system. A. awamori efficiently consumed starch in wheat bran medium and produced very high glucoamylase activity, and after that, the fungus efficiently produced other enzymes to degrade other complex nutrients such as protein, hemicellulose and cellulose. Meanwhile, $A$. oryzae efficiently consumed protein in rapeseed meal and produced very high protease activity. The ability of both filamentous fungi, to convert biomass through SSF bioconversion will have a great impact on food and agro-industry in every aspect of life from food and medicine to fuel.
\end{abstract}

Key words: Tray system, solid state fermentation, Aspergillus awamori, Aspergillus oryzae, glucoamylase, protease, xylanase, cellulase.

\section{Introduction}

Solid state fermentation (SSF) processes play an important role in the production of various microbial enzymes. The production of enzymes by SSF is associated with the history of the development of SSF and enzymes have been known to be produced by SSF for many years [1]. Enzymes have been established to be useful due to their wide range of applications. Enzymes have tremendous applications in a number of industrial processes such as the food industry, fermentation, the textile industry and the production

Corresponding author: Colin Webb, Ph.D., professor, research field: microbial fermentation and bioprocess. of paper and also in chemical feedstocks. Therefore, there is an increasing demand for their production [2]. Although, all commercial production of enzymes as well as almost all research works on microbial enzymes employs submerged fermentation (SmF) as the technique of fermentation, SSF has also been successfully used to cultivate many microorganisms using various solid substrates.

SSF offers several potential advantages for the production of enzymes. It has been well established that enzyme titres produced in SSF are many fold more concentrated than those produced by $\mathrm{SmF}$. George et al. [3] reported that the total protease activity present in one gram wheat bran (SSF) was 
equivalent to that present in $100.0 \mathrm{~mL}$ broth $(\mathrm{SmF})$. In addition, SSF can be of special interest in those processes where the crude fermented product may be used directly as a source for enzymes [3]. The enzymes in SSF crude fermented product are concentrated; thus, they can be used directly in such agro-biotechnology applications as silage or feed additives, ligno-cellulosic hydrolysis and natural fibre processing [4].

The selection of an excellent microorganism strain for the production of enzymes is very important. The microorganism selected, especially the fungus in this case, must have relatively stable characteristics and the ability to grow rapidly and vigorously [5]. The most important characteristic to look for in the selection of the microorganism is its ability to degrade the complex solid substrates. This will lead to the production of higher yields of the desired enzymes. The selection of a particular strain of microorganism, however, remains a tedious task, especially when commercially competent enzyme yields are to be achieved $[6,7]$. This will be dependent upon a number of factors, mainly including the nature of substrate and the environmental conditions.

Due to the lack of free water in SSF, smaller fermenters are required for this process, and therefore, less effort is required for downstream processing (van Breukelen et al., 2011). Tray systems are the simplest of all types of fermenters used in SSF. As has been mentioned in open literature, there are several other types of designs of fermenters that have been applied by researchers for various purposes. The bed height of a tray reactor is limited due to heat and mass transfer limitations and heat transfer gradients [8]. By contrast, rotary reactors are deemed to have complex operational systems, low fill volumes (typically less than 30\%) and low shear in the substrate bed [9].

In this work, studies were carried out in a simple tray system, which was used as a closed system or an opened system. For the closed system, normal petri dishes were used. For the opened system, a circular stainless steel tray was used. The bottom of the opened tray is perforated (with different aperture sizes) in such a way that it holds the substrate and allows aeration. This system was designed to address the current problems in SSF and to ensure consistent water content in the fermented solid substrate during fermentation. The study tested fermentation with Aspergillus awamori and Aspergillus oryzae and monitored the efficiency of multi-enzyme production (glucoamylase, protease, xylanase and cellulose).

\section{Materials and Methods}

\subsection{Microorganisms and Preparation of the Inoculum}

The fungi A. awamori and A. oryzae were used to study enzyme production. The spores were washed by lightly scrapping with wire loop in $10.0 \mathrm{~mL}$ of sterile $0.1 \%(\mathrm{v} / \mathrm{v})$ Tween $80.0 .5 \mathrm{~mL}$ of the spore suspension was further transferred onto the surface of $100.0 \mathrm{~mL}$ of sporulation medium in $500.0 \mathrm{~mL}$ Erlenmeyer flask and incubated for another 7 days at $30^{\circ} \mathrm{C}$. After the incubation period, $50.0 \mathrm{~mL}$ of sterile $0.1 \%(\mathrm{v} / \mathrm{v})$ Tween 80 solution and several sterile glass beads (4-mm diameter) were added to the flask. The spores were suspended by shaking the flask gently and collected in one bottle as a spore suspension. The concentration of the spore suspension was measured by haemocytometer. The volume of suspension needed for the inoculation of the solid was calculated for each experiment to reach a concentration of around $1.0 \times 10^{6} \mathrm{spores} / \mathrm{g}$ solid substrate. Fungal spores in universal bottle were stored at $4{ }^{\circ} \mathrm{C}$ in agar slopes of solid sporulation medium containing $5 \%(\mathrm{w} / \mathrm{v})$ whole wheat flour and $2 \%(\mathrm{w} / \mathrm{v})$ agar as a stock culture.

\subsection{The Substrates-Solid State Fermentation}

The solid substrates: wheat bran, soybean hulls and rapeseed meal, were used. Wheat bran was obtained from Cargill Wheat Processing Plant, Manchester, UK. Soybean hulls and rapeseed meal were obtained from Brocklebank Oilseed Processing Division, Cargill Wheat Processing Plant, Liverpool, UK. All three 
selected substrates were used without any treatment as a solid medium for growing A. awamori and A. oryzae. At time intervals, sample was taken out. The performance of the systems was evaluated in terms of the production of enzymes regards to fungal growth.

\subsection{The Tray System}

Two types of tray systems, known as a closed or opened system, were used in this study. A closed system consists of normal petri dishes with $9 \mathrm{~cm}$ diameter and $1.5 \mathrm{~cm}$ depth covered with a lid. The opened system consists of circular stainless steel trays with $10 \mathrm{~cm}$ diameter and $3.5 \mathrm{~cm}$ depth. The top of the tray is covered with a lid and the bottom of the tray is equipped with a perforated base to allow for aeration of the bottom surface of the bed and, to some extent, the inner layers within the bed.

As an opened system, the aperture size of perforated base was $45,53,106,180,300,425,500,600,710$, $850,1000,1400,2000$ or $2800 \mu \mathrm{m}$. These trays are autoclave compatible. $12.0 \mathrm{~g}$ of wheat bran and soybean hulls and $15.0 \mathrm{~g}$ of rapeseed meal were used with both systems. These amounts were used to bring the height of the substrate bed in the tray to $1.0 \mathrm{~cm}$. After autoclaving, inoculum transfer was carried out aseptically and sterile distilled water was used to moisten the solid substrates. The initial moisture content of the substrate was $65 \%$. After the inoculation process, all the trays were placed in a growth incubator with temperature strictly under control at $30{ }^{\circ} \mathrm{C}$. At the end of the incubation period $(72 \mathrm{~h})$, the performance of the two systems was evaluated in terms of the production of four enzymes, which are glucoamylase, protease, xylanase and cellulase.

\subsection{Preparation of Enzymes Supernatant}

After the $72 \mathrm{~h}$ fermentation period, samples were taken for enzyme analysis. All the fermented samples ( $2.0 \mathrm{~g}$ on a wet basis) were extracted with distilled water $(40.0 \mathrm{~mL})$ and shaken for $30 \mathrm{~min}$ on a rotary shaker (Infors A-CH 4103 Switzerland) at $250 \mathrm{rpm}$ and $30{ }^{\circ} \mathrm{C}$. Then the solid suspensions were centrifuged at $10,000 \mathrm{rpm}$ for $10 \mathrm{~min}\left(4^{\circ} \mathrm{C}\right)$. The clear supernatant was used for the measurement of enzyme activity. A standard operational procedure for enzyme extraction developed in this study. A normal observation in SSF is un-even growth on fermented substrate. This situation will usually result in unsatisfied outcome. Therefore, a standard procedure was developed in this study to obtain a satisfactory sampling process. To overcome this issue and to satisfy the need for a homogenous sampling process, an amount of fermented mass was harvested, crashed and vigorously mix using spatula. If the experiments were conducted in petri dishes, the whole fermented mass was blended using a food-processing blender.

\subsection{Enzymes}

\subsubsection{Glucoamylase Activity}

Glucoamylase was assayed using the method as described by Ariff and Webb [10] using maltose as a substrate. Glucoamylase activity was determined by measuring the initial rates of glucose production and expresses as $\mu$ mole of glucose liberated per minute per $\mathrm{mL}$ broth supernatant $\left(\mu\right.$ mole $\mathrm{min}^{-1} \mathrm{~mL}^{-1}$ ) or unit per $\mathrm{mL}(\mathrm{U} / \mathrm{mL})$. Glucoamylase activity is expressed throughout this study in units of $\mathrm{U} / \mathrm{g}$ material measured on a dry basis.

\subsubsection{Protease Activity}

Protease activity was evaluated by the formation of free amino nitrogen (FAN). FAN concentration was measured using the ninhydrin colourimetric method as outlined by the European Brewery Convention [11] with modifications made by Wang [12]. The method based on the colour reaction between ninhydrin and amino acids at $\mathrm{pH}$ 6.7, which gives an estimate of amino acids, ammonia and in addition the terminal alpha-amino nitrogen groups of peptides and proteins. The amount of proteases for the production of one milligram FAN in one minute under controlled conditions was defined as one unit of activity $(\mathrm{U} / \mathrm{mL})$. The protease activity throughout this study was 
expressed in units $\mathrm{U} / \mathrm{g}$ material measured on a dry basis.

\subsubsection{Xylanase Activity}

Determination of xylanase activity was conducted according to the method developed by Bailey et al. [13]. The assay is based on the release of reducing sugars from $1 \%(\mathrm{w} / \mathrm{v})$ xylan (Sigma-Aldrich) solution prepared in $0.05 \mathrm{M}$ citrate buffer $\mathrm{pH} 5.4$ by 3,5-dinitrosalycylic acid method (DNS method) at $50{ }^{\circ} \mathrm{C}$ by using xylose to generate a standard curve [14]. The xylanase activity was expressed throughout this work in units of $\mathrm{U} / \mathrm{g}$ material measured on dry basis. One unit of xylanase activity was defined as the amount of enzyme producing $1 \mu$ mole xylose equivalents per minute under assay conditions.

\subsubsection{Cellulase Activity}

Filter paper cellulase activity was measured according to IUPAC recommendations employing filter paper Whatman No. 1 as a substrate. The procedures were designed to measure cellulase activity in terms of "filter paper units" (FPU) per millilitre $(\mathrm{mL})$ of original (undiluted) enzyme solution. Quantitative results of the enzymes preparations must be compared on the basis of significant and equal conversion. The value $2.0 \mathrm{mg}$ of total reducing sugars as glucose from $50.0 \mathrm{mg}$ of filter paper in $60 \mathrm{~min}$ reaction time was designated as the intercept for calculating filter paper cellulase units (FPU) by IUPAC. The total reducing sugars was then determined by the DNS method [14]. The cellulase activity was expressed throughout this study in units of FPU/g material measured on a dry basis.

\section{Results and Discussion}

Food and agro-industry solid waste residues are generally considered the best substrates for SSF processes and enzyme production in SSF in terms of nutritional value [15]. These substrates, which are derived from various sources, vary in their nature, structure and composition. The quality of substrates differs due to their composition and the ratios of different ingredients. These include starch, protein, lignin, cellulose and hemicellulose. Therefore, cultures from different strains of microorganisms biodegrade substrates in varied fashions and produce different enzymes, such as glucoamylase, protease, xylanase and cellulase. In the end product of fermentation, enzymes should be present in a heterogeneous mixture as a result of the metabolic activity of the employed microorganism [16].

Preliminary results show that there are no significance differences in the values obtained for enzyme production in the opened system with different aperture sizes (perforated base aperture size $45,53,106,180,300,425,500,600,710,850,1000$, 1400, 2000 and $2800 \mu \mathrm{m})$. Thus, in this article, the values obtained using the opened system will be presented as an average for the different measurements corresponding to aperture sizes ranging from 45 to $2800 \mu \mathrm{m}$. The data are compared with those from the closed system to investigate the performance differences between the two systems.

\subsection{Enzymes Production in SSF using the Tray System}

Many researchers have reviewed the production of enzymes in various modified solid state tray systems. Nahid et al. [17] reviewed glucoamylase production and Mitra et al. [18] reviewed the production of proteolytic enzymes. Babu and Satranayanam [19] examined bacterial enzyme production, while Gupta and Kar [20] reviewed the production of cellulolytic enzymes. In addition, Dhillon et al. [21] reported the production of cellulase and xylanase enzymes in tray SSF that employed mixed-cultured fungi.

\subsubsection{Glucoamylase}

Activity levels for glucoamylase that was produced in the two systems are shown in Figure 1. Glucoamylase activity levels of 299.47, 88.74 and $75.96 \mathrm{U} / \mathrm{g}$ [db] were obtained after $72 \mathrm{~h}$ of fermentation time in the closed system with $A$. awamori for wheat bran, soybean hulls and rapeseed meal respectively. A. awamori in the opened system 
produced an average glucoamylase activity of 336.08 , 90.92 and $32.82 \mathrm{U} / \mathrm{g}[\mathrm{db}]$ obtained for wheat bran, soybean hulls and rapeseed meal respectively. Glucoamylase activity levels were higher when SSF was carried out in the opened system with wheat bran and soybean hulls. This was attributed to the efficient air circulation and nutrient diffusion caused by the perforated base resulting in an increase in $\mathrm{O}_{2}$ and mass transfer. However, almost a two-fold glucoamylase activity decrease was recorded for rapeseed meal culture in the opened system compared to the closed system.

In addition, glucoamylase activity levels of 121.62, 85.96 and $79.06 \mathrm{U} / \mathrm{g}[\mathrm{db}]$ were obtained after $72 \mathrm{~h}$ of fermentation time in the closed system with $A$. oryzae for wheat bran, soybean hulls and rapeseed meal respectively. A. oryzae in the opened system produced an average glucoamylase activity of 81.30, 76.94 and $57.30 \mathrm{U} / \mathrm{g}[\mathrm{db}]$ obtained for wheat bran, soybean hulls and rapeseed meal, respectively. This is a contrast to the result obtained with $A$. awamori. Glucoamylase activity decreased in the opened system almost 1.5 times in A. oryzae culture on rapeseed meal compared to the closed system.

A. awamori produced high glucoamylase levels in the opened and the closed system respectively. A. awamori favoured growth on wheat bran and produced high glucoamylase activity on this substrate. Higher enzyme production on wheat bran can be

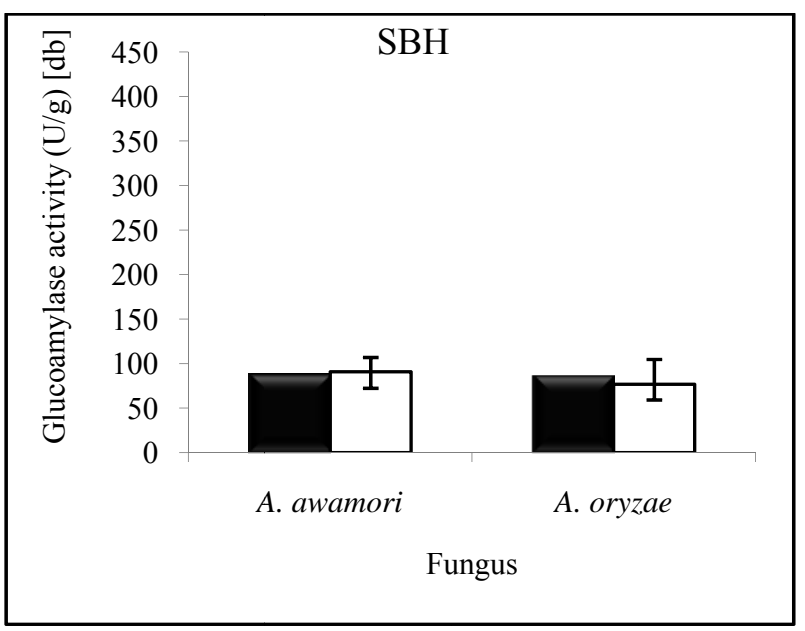

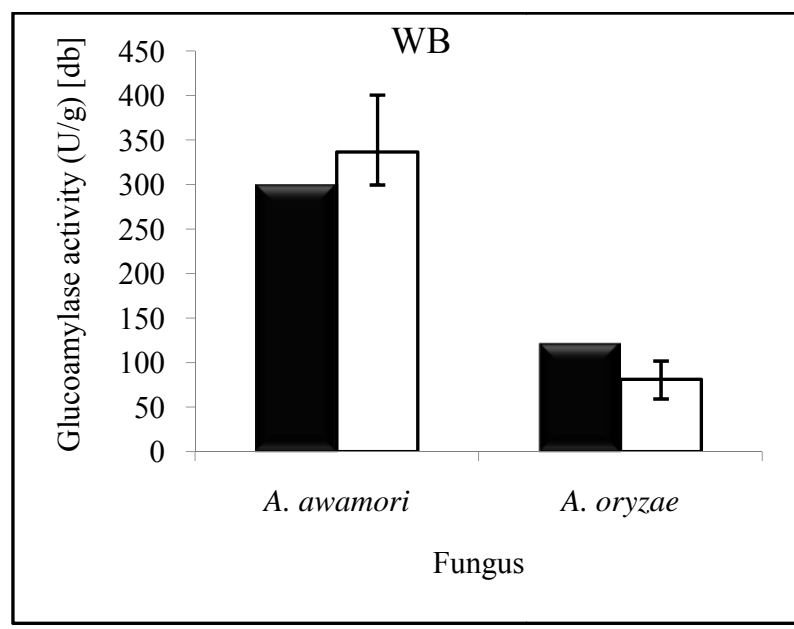

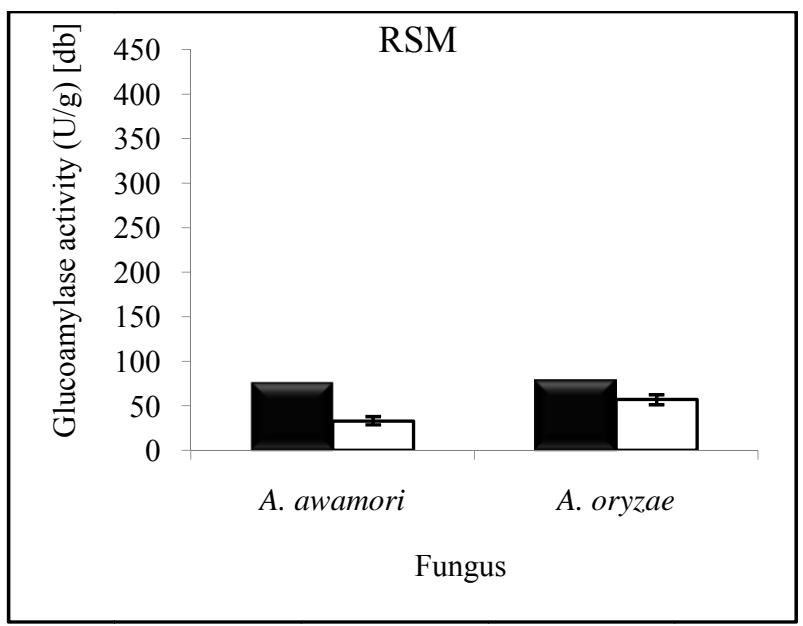

Fig. 1 Activity of glucoamylase produced in the closed $(\square)$ and the opened system $(\square)$ by the fungal culture of $A$. awamori and $A$. oryzae on different solid substrates. [WB]: wheat bran; [SBH]: soybean hulls and [RSM]: rapeseed meal. Data points show the average of measurements for the opened tray system with 14 different mesh aperture sizes. 
correlated with its high starch content (23.3\%) [22]. Nahid et al., 2011 induced production of glucoamylase $(373.3 \mathrm{IU} / \mathrm{g})$ in wheat bran when it combination with corn flour compared to wheat bran alone. However, A. oryzae does not favoured on wheat bran. A. awamori with wheat bran media resulted in double the enzyme production by A. oryzae culture.

Ellaiah et al. [23] reported that wheat bran gives higher enzyme yield compared to rice bran and other agricultural waste. Hata et al. [24] compared glucoamylase levels produced in SSF and SmF systems using $A$. oryzae and showed these systems exhibited different characteristics. In their work, they reported that glucoamylase produced by SSF could digest raw starch but that produced by $\mathrm{SmF}$ could not. Pandey [25] reported that glucoamylase production in trays occurred optimally after $36 \mathrm{~h}$ of fermentation in comparison to the typically required $96 \mathrm{~h}$ periods in flask fermentation processes.

\subsubsection{Protease}

Protease activity levels measured in samples in the studied systems are shown in Figure 2. Protease activity levels of 93.08, 42.05 and $40.04 \mathrm{U} / \mathrm{g}$ [db] were obtained after $72 \mathrm{~h}$ of fermentation time in the closed system with A. awamori for rapeseed meal, soybean hulls and wheat bran respectively. However, A. awamori in the opened system produced an average protease activity of $165.03,114.92$ and $70.32 \mathrm{U} / \mathrm{g}$ [db] obtained from rapeseed meal, soybean hulls and wheat bran, respectively. Protease activity increased about two-fold when SSF was carried out in the opened system compared to the closed system.

Meanwhile, protease activity levels of 1017.70, 972.35 and $964.71 \mathrm{U} / \mathrm{g}$ [db] were obtained after $72 \mathrm{~h}$ of fermentation time in the closed system with $A$. oryzae for rapeseed meal, soybean hulls and wheat bran, respectively. In contrast to the closed system, A. oryzae in the opened system produced an average protease activity of 2029.51, 919.50 and $1327.76 \mathrm{U} / \mathrm{g}$ [db] obtained from rapeseed meal, soybean hulls and wheat bran, respectively. In line with observations with A. awamori, protease activity increased almost two-fold in the opened system compared to the closed system.

These results proved that $A$. oryzae is an excellent fungus for protease production with rapeseed meal as a solid substrate. This is expected since rapeseed meal content is higher in protein content $(38.9 \%)$ than wheat bran (15.1\%) and soybean hulls (14.2\%). In this case, the opened system proved to be more favourable for protease production for both fungi. Improvement of mass transfer in the opened system might be a reason explaining this behaviour. Another can be low temperature and moisture content of the fermented solid that may be beneficial and may cause efficient degradation of substrate and uptake of nutrients. The decrease in moisture content in the opened system, especially with $A$. oryzae culture, did not reach a critical point that can inhibit growth. Protease produced by $A$. oryzae was efficient in breaking down the peptide bonds of proteins contained in rapeseed meal, and hence, forming more FAN.

George et al. [3] reported that one gram of wheat bran was found to produce about $250,000 \mathrm{U} / \mathrm{mL}$ protease activity in SSF using B. amyloliquefaciens by subjecting the microorganism to natural selection and high positive organic nitrogen pressure. Meanwhile, Belmessikh et al. [26] reported that protease activity was nine times higher in $\operatorname{SSF}(21,309 \mathrm{U} / \mathrm{g})$ compared to $\mathrm{SmF}(2343.5 \mathrm{U} / \mathrm{g})$ using $A$. oryzae on tomato pomace.

\subsubsection{Xylanase}

Xylan is a polysaccharide found in the hemicellulose fraction of lignocellulose. Hemicellulose of agricultural substrate can be used as an appropriate substrate for xylanase production in SSF [27]. Xylanase hydrolyses xylan to xylose or xylooligosccharide. Activity levels for xylanase extracted in both studied systems with A. awamori and A. oryzae are shown in Figure 3.

Maximum xylanase activity levels of 728.24 , 586.17 and $450.63 \mathrm{U} / \mathrm{g}$ [db] were obtained after $72 \mathrm{~h}$ 


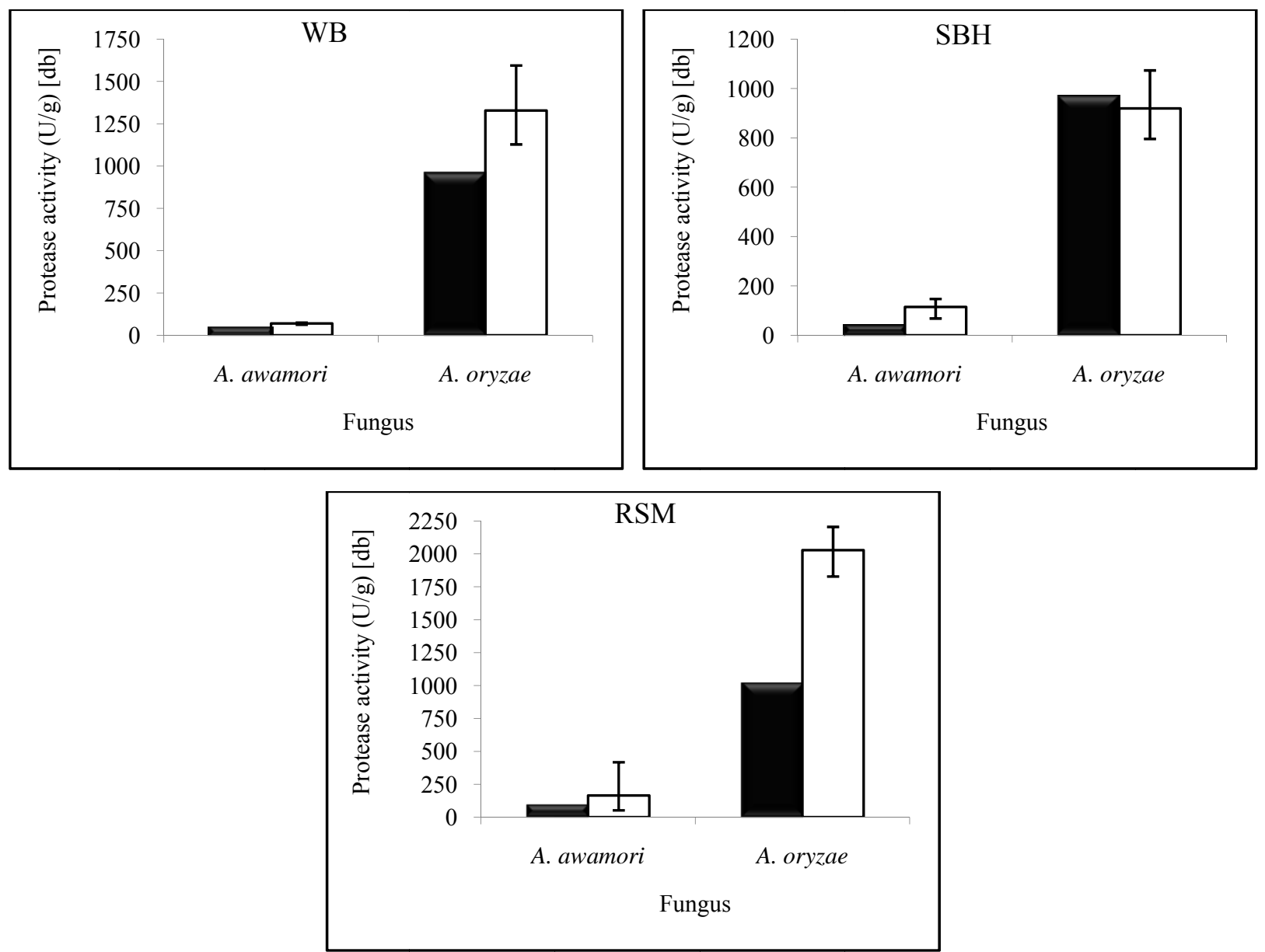

Fig. 2 Activity of protease produced in the closed $(\square)$ and opened system ( $\square$ ) by the fungal culture of A. awamori and A. oryzae on different solid substrates. [WB]: wheat bran; [SBH]: soybean hulls and [RSM]: rapeseed meal. Data points show the average of measurements for the opened tray system with 14 different mesh aperture sizes.

of fermentation time in the closed system with A. awamori for wheat bran, soybean hulls and rapeseed meal respectively. However, A. awamori in the opened system produced an average xylanase activity of $581.42,389.65$ and $349.49 \mathrm{U} / \mathrm{g}$ [db] obtained for wheat bran, soybean hulls and rapeseed meal, respectively. There were significant differences between the closed and opened system for all reported activities. A. awamori was observed to be superior in producing xylanase in the closed system compared to the opened system. This observation can be related to the increase in moisture content in the closed system, which may favour xylanase production. It was reported that the optimum moisture content for xylanase production by T. longibrachiatum [28] and $A$. tereus [29] on wheat bran were 55 and $75 \%$ respectively. Production of xylanase decreased in the opened system, which might be related to loss of moisture content and subsequent inhibition of $A$. awamori growth.

Maximum xylanase activity levels of 103.94, 52.71 and $17.14 \mathrm{U} / \mathrm{g}$ [db] were obtained after $72 \mathrm{~h}$ of fermentation time in the closed system with A. oryzae for wheat bran, soybean hulls and rapeseed meal, respectively. However, A. oryzae in the opened system showed an increase in xylanase activity to an average of $219.71,138.71$ and $117.87 \mathrm{U} / \mathrm{g}$ [db] observed for soybean hulls, wheat bran and rapeseed meal, respectively. In contrast to the results obtained for $A$. awamori above, the production of xylanase was higher in the opened system compared to the closed system. 
Significant differences were observed in the produced xylanase activity between A. awamori and A. oryzae in both systems. In the closed system with $A$. awamori on wheat bran, xylanase production was 7 times higher compared to A. oryzae in the same system. Production of xylanase seemed very poor with A. oryzae in both systems with all three substrates tested. These findings show that A. awamori is efficient in producing xylanase and in actively degrading the bonds of hemicellulose contained in wheat bran, soybean hulls and rapeseed meal and forming more reducing sugars. This is another reason TRS was higher in A. awamori culture. High activities were obtained for wheat bran followed by soybean hulls, both of which contained a high proportion of hemicellulose (29.7\% and $12.5 \%$, respectively) [22] and therefore induced xylanase production. Rapeseed meal, however, has lower hemicellulose content [16]. Hemicellulose of agricultural substrate can be used as an appropriate substrate for xylanase production in SSF [27]. However, A. oryzae is observed to be very poor in the production of xylanase.

Observations of this work indicate the production of xylanase is higher in a closed system. In the closed system, it was assumed that heat accumulation occurred and therefore incubation temperature increased and subsequently the production of xylanase. This phenomenon was also observed in glucoamylase production (Figure 1). Cai et al. [27] reported production of thermostable xylanase in an SSF system using A. niger $\mathrm{A} 3$ and this enzyme was more thermostable $\left(55^{\circ} \mathrm{C}\right)$ than that produced in $\mathrm{SmF}$.
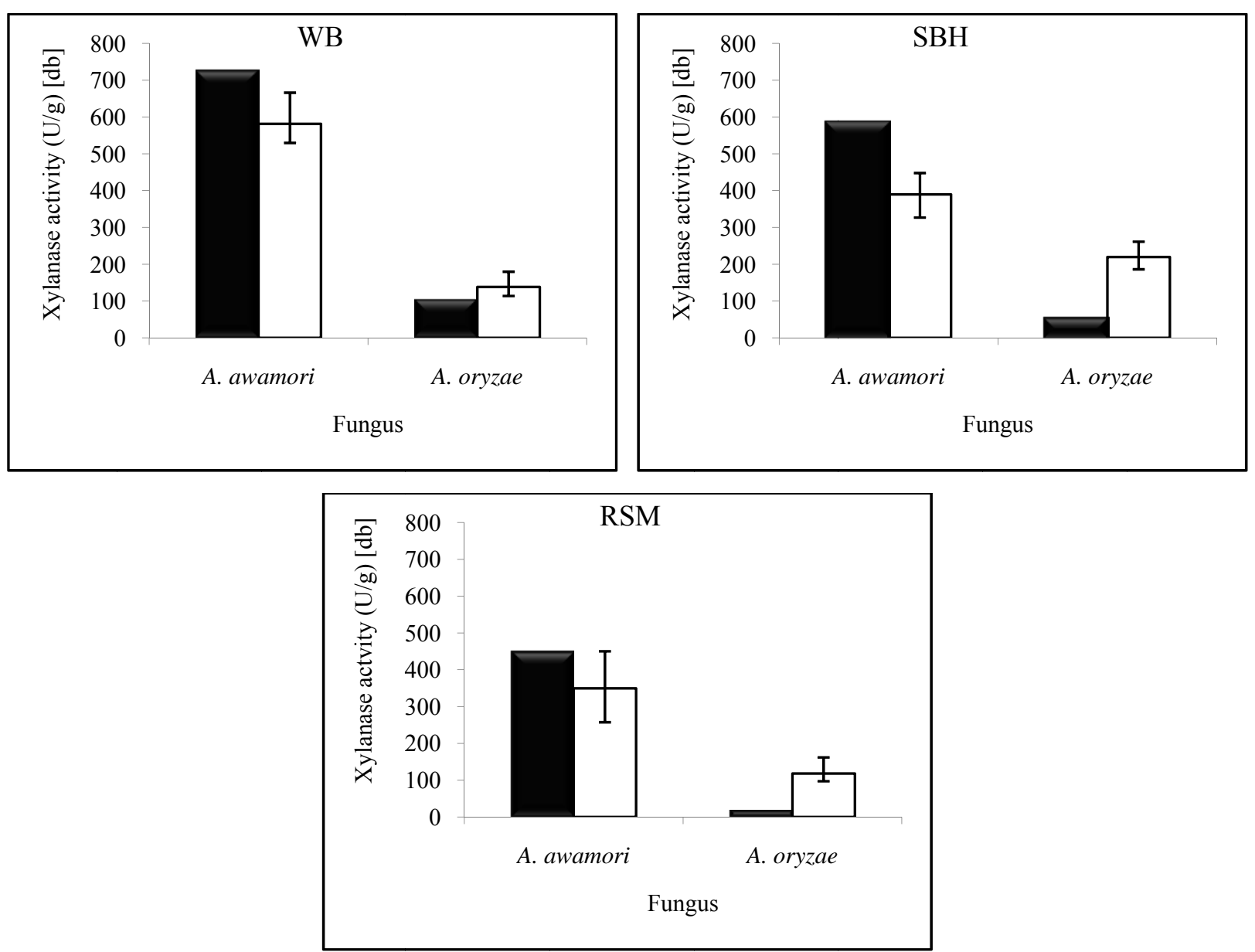

Fig. 3 Activity of xylanase produced in the closed $(\square)$ and opened system ( $\square$ ) by the fungal culture of $A$. awamori and $A$. oryzae on different solid substrates. [WB]: wheat bran; [SBH]: soybean hulls and [RSM]: rapeseed meal. Data points show the average of measurements for the opened tray system with 14 different mesh aperture sizes. 
Archana and Satyanarayana [30] also reported production of cellulase-free xylanase at levels 22 fold higher in SSF compared to SmF using thermophile $B$. licheniformis A99. In addition, the SSF process was also observed to induce production of higher levels of xylanase by thermostable Melanocarpus albomyces due to the close contact between the mycelium and the solid substrate compared to $\mathrm{SmF}$ [31]. According to Pandey et al. [4], although xylanase is produced by fungi, yeast and bacteria, filamentous fungi are preferred for commercial production as the levels of enzyme produced by fungal cultures are higher than those obtained from yeast or bacteria.

\subsubsection{Cellulase}

Cellulases are among the most important enzymes that are employed in the processing of ligno-cellulosic materials for the production of feed, fuel and chemical feedstocks [32-34]. Activity levels of cellulase involved in both studied systems were measured using an enzyme extract that was prepared after fermentation with A. awamori and A. oryzae (Figure 4).

Maximum filter paper activity levels of 17.28 , 16.99 and 16.08 FPU/g [db] were obtained after $72 \mathrm{~h}$ of fermentation time in the closed system with $A$. awamori for wheat bran, rapeseed meal and soybean hulls, respectively. However, A. awamori in the opened system produced an average filter paper activity level of 14.03, 10.19 and $8.19 \mathrm{FPU} / \mathrm{g}$ [db] obtained for soybean hulls, rapeseed meal and wheat bran, respectively. There was no significant difference between solid substrates especially in the closed system.
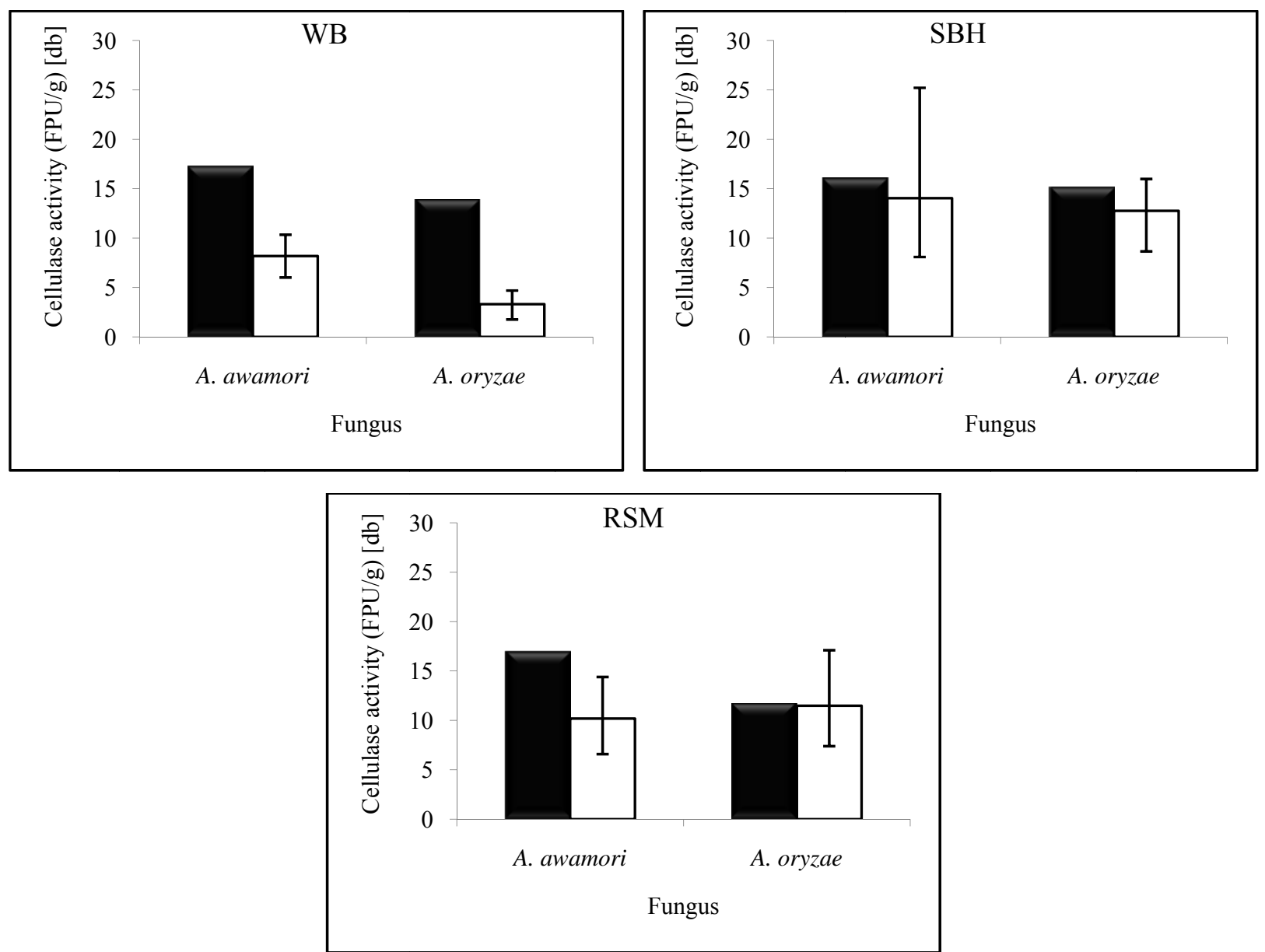

Fig. 4 Activity of cellulase produced in the closed ( $\square$ ) and opened system ( $\square$ ) by the fungal culture of A. awamori and A. oryzae on different solid substrates. [WB]: wheat bran; [SBH]: soybean hulls and [RSM]: rapeseed meal. Data points show the average of measurements for the opened tray system with 14 different mesh aperture sizes. 
Maximum filter paper activity levels of 15.18 , 13.88 and 11.67 were obtained in the closed system with A. oryzae for soybean hulls, wheat bran and rapeseed meal, respectively. In the opened system, an average filter paper activity level of 12.57, 11.48 and $3.32 \mathrm{FPU} / \mathrm{g}[\mathrm{db}]$ was obtained for soybean hulls, rapeseed meal and wheat bran, respectively.

A. awamori was observed to be superior in producing cellulase on wheat bran and soybean hulls in the closed and opened system. A. oryzae was observed to be excellent in producing cellulase on soybean hulls in both systems compared to the other two substrates. Cellulose content in soybean hulls (36.4\%) [35] was obviously higher than that in wheat bran (10.6\%) [22], and as expected, cellulose is ideal for efficient growth of fungal cultures and cellulase production. The results of static tray SSF highlighted the importance of using the closed or the opened system. Overall, cellulase production was significantly higher in closed system SSF as compared to the opened system for both fungi. Production of cellulase decreased in the open system for both fungi, which might be due to the drastic loss of moisture content in this system.

Brijwani et al. [36, 37] reported that SSF of soybean hulls supplemented with wheat bran produced a maximum filter paper activity of 10.7 FPU/g [db] after a $96 \mathrm{~h}$ incubation period in a static tray. According to Amorea Faracoa [38], only fungi naturally produce the needed titers of cellulases required for the complete saccharification of pretreated lignocellulose. Cellulolytic fungi (T. reesei, Aspergillus spp., Fusarium spp., Rhizopus spp.) including thermophilic fungi, such as T. emersonii and T. aurantiacus, produce a high concentration of cellulase enzymes to digest lignocellulose efficiently, assimilate all ligno-cellulosic sugars and convert these sugars to ethanol, showing that they naturally possess all pathways for the conversion of lignocellulose to bioethanol [39]. The efficiencies of the degradation of cellulose were dependent on the nature and concentration of the compounds added in the SSF system. The production of fermentative sugars as a result from the hydrolysis of biomass component (hemicellulose and cellulose) is largest economic and technological barrier for the production of cellulosic biofuels [40].

\subsection{Mass Transfer Phenomenon in the Tray System}

In this work, it was observed that A. awamori and A. oryzae react differently to the design applied in the tray system. There were also differences between of $A$. awamori and A. oryzae in the degradation of the solid substrates tested. These differences led to variations in growth performance and production of enzymes. A. awamori favoured the opened system for glucoamylase production, while A. oryzae favoured the closed system. Glucoamylase production increased when access to $\mathrm{O}_{2}$ was improved in the opened system using a perforated tray compared to the closed system. Xylanase and cellulase production was observed to be superior in the closed system with A. awamori, and protease production was excellent in the opened system with A. oryzae.

From visual observation (in the case of A. awamori), the morphology growth in the closed and opened systems shows some difference as shown in Figure 5. The same phenomenon was observed for A. oryzae. In this work, two types of mycelia were observed in the vegetative growth of both fungi. First, the growth penetrating the surface of the solid substrate leads to the formation of penetrated mycelium. Second, the aerial growth leads to the formation of aerial mycelia. However, it is difficult to differentiate between these mycelia on the surface. According to Sudo et al. [41], after germination, the penetrated mycelium grows first, and then aerial mycelium develops above it. The sporangiophores that produce the conidiospores are formed later within the aerial mycelia. Additionally, Sudo et al. [41] studied differences between these two types of mycelium in terms of the production of $\alpha$-amylase in rice koji. They observed that $\alpha$-amylase 


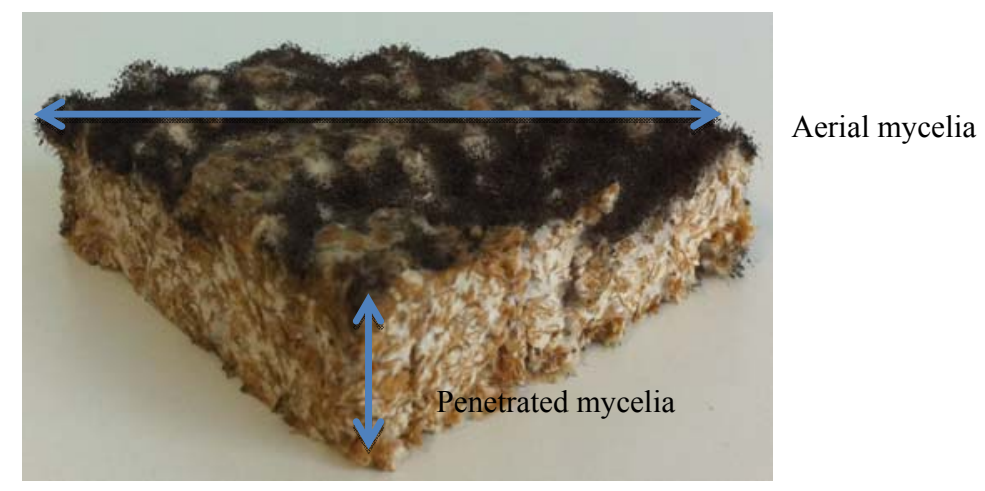

(a)

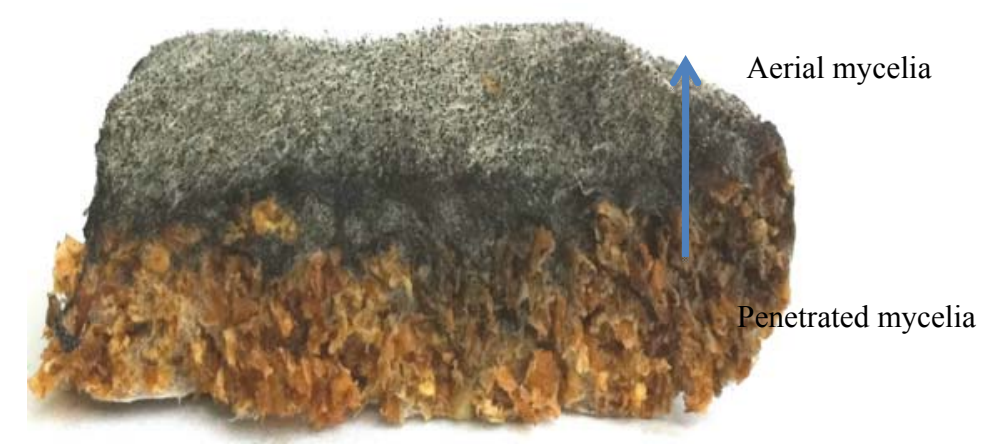

(b)

Fig 5 Images of the fermented cake of $A$. awamori on wheat bran in (a) the closed system and (b) the opened system.

was found to be produced highly by the penetrated mycelium rather than the aerial mycelium.

Figure 5(a) shows the mycelium penetrates deeply into the inner solid substrate particles of wheat bran. The deep penetrating clean white mycelium and binding together the solid substrate, covered almost every part of the fermented bed and produced a compact fermented cake. Figure 5(b) shows the large aerial mycelium on the surface of the fermented bed, showing sporangiophores, which would produce large spores. The fermented cake observed is quite loose and porous from the middle of the fermented cake to the bottom surface of the perforated base. The production of mycelium in the open system was lower compared to the closed system. It was assumed that, in the area exposed to the environment, the concentration of $\mathrm{O}_{2}$ is very high yet moisture content is low. This will lead to lower diffusivity of glucose and other nutrients into solid particles of solid substrates of low moisture content. These results mean that the production of glucose and other nutrients is restricted at low moisture content and any glucose and other nutrients available are consumed rapidly before they diffuse along the particles. As a result, some starvation may occur on solid substrates of low moisture content, which may affect growth performance. Therefore, it was concluded that moisture content in the fermented substrate is one of the most important factors for the production of enzymes in SSF.

Figure 6(a) shows the closed non-aerated system showing the advantages of tray SSF; it allows for the strict control of water content in the substrate during culture by allowing internal vapour condensation. $\mathrm{O}_{2}$ concentration in the system might be low, but this disadvantage might be overcome where fungi are totally dependent on water content to support growth. Internal vapour condensation might become detrimental because it can create non-homogenous conditions. Fungi grew more vigorously in the cultures with higher water content. It was observed 
from the study in the lab that moisture content in the closed non-aerated system could be maintained for up to $200 \mathrm{~h}$ of fermentation time. Consequently, the increase in moisture content of the fermented bed could be cause-decreased porosity, change in particle structure and lower $\mathrm{O}_{2}$ transfer in the solid substrate bed. The moisture content in the closed system was assumed to be reasonable between $65-73 \%$.

As in the opened system (Figure 6(b)), the contribution of water evaporation as a consequence of metabolic heat production might not happen because water evaporation occurs at the perforated base. No water accumulation is observed. These results indicated that the vapour generated from microbial activity could efficiently dissipate out of the tray through the perforated base. Nevertheless, the total amount of evaporated water was estimated to be higher in the opened system. Water evaporation depends on the amount of metabolic heat evolved, so it can be suggested that for the opened system, the heat evolved reduces the moisture content of the solid substrate by evaporation in the case of both fungi.

One of the important roles and functions of the fungi in SSF is the synthesis of enzymes, in particular extracellular enzymes. These enzymes generally hydrolyse complex compounds such as starch, proteins, and polysaccharides into smaller molecules, which in turn can be taken up by any cell. As a consequence, the reduction of moisture content leads to reduce diffusion of enzymes and nutrients in the fermented substrate, lower degree of swelling and increased water tension on the microorganism.

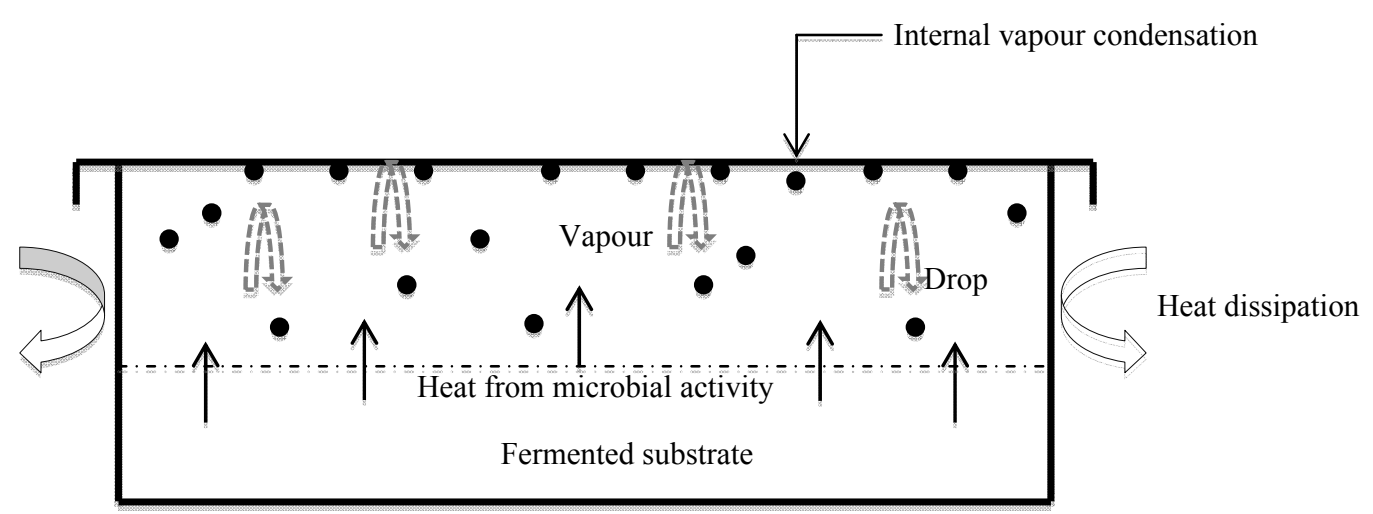

(a)

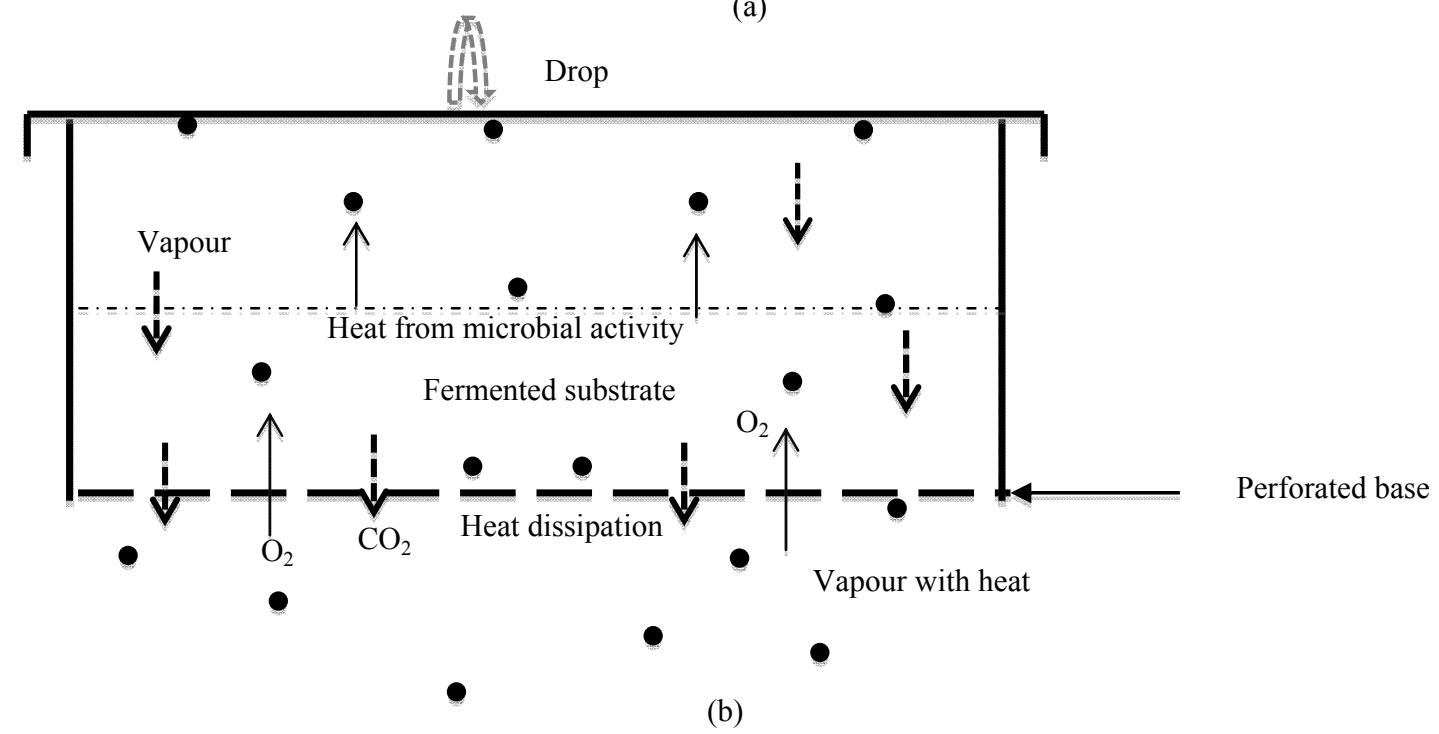

(b)

Fig. 6 Schematic cross-sectional diagrams of the (a) closed system and (b) opened system. Briefly, phenomenon mass transfer during fermentation process. 


\section{Conclusions}

From this work, the following conclusions can be made with regards to the systems used; the closed system (i) $\mathrm{O}_{2}$ concentration is a limiting factor that influences fungal growth and (ii) the ability to maintain water is high but it creates heterogeneous conditions. The opened system; (i) $\mathrm{O}_{2}$ concentration might not be a problem, but the drastic loss of moisture content of about $80 \%$ (especially in the culture system with A. awamori) after $72 \mathrm{~h}$ of fermentation is a limiting factor that influences fungal growth and (ii) the chance to lose water through evaporation is very high through the uncontrolled perforated base tray.

It is possible that the opened system exhibits such a high reproducibility of enzyme production because it allows efficient mass transfer and heat dissipation, although it is also observed to be more efficient in evaporation of water from the substrate. SSF in the closed system faces difficulty in controlling these parameters because it is a closed environment. On top of that, it is difficult to achieve reproducible results under appropriate culture conditions because both $A$. awamori and $A$. oryzae respond differently with varied growth performances. This can be observed to be reflected in enzyme production. However, the results obtained in this work provide an idea of how to make an air arrangement into the closed solid state tray bioreactor system. The important idea is that it is possible to achieve uniform and exact regulation of temperature and water content during the fermentation process without introducing unfavourable factors such as substrate mixing. Accumulation of high amylolytic and proteolytic enzymes observed in this study is desired as this will result in a successful hydrolysis process that follows the process of SSF and targets the production of a medium rich in fermentative sugars and nitrogen. Through a strategy for a biorefinery based on SSF, this can lead to the production of bioethanol, biofuels, biodegradable plastic and valuable chemicals.

\section{References}

[1] Pandey, A. 1992. "Recent Process Developments in Solid State Fermentation." Process Biochemistry 27: 109-117.

[2] Rodriguez-Fernandez, D. E., Rodriguez-Leon, J. A., de Carvalho, J. C., Sturm, W., and Soccol, C. R. 2011. "The Behaviour of Kinetic Parameters in Production of Pectinase and Xylanase by Solid-State Fermentation." Bioresource Technology 102: 10657-662.

[3] George, S., Raju, V., Subramaniam, T. V., and Jayaraman, K. 1997. "Comparative Study of Protease Production in Solid substrate Fermentation versus Submerged Fermentation." Bioprocess Engineering 16: 381-2.

[4] Pandey, A., Selvakumar, P., Soccol, C. R., and Nigam, P. 1999. "Solid State Fermentation for the Production of Industrial Enzymes." Current Science 77 (1): 149-62.

[5] Ito, K., Kawase, T., Sammoto, H., Gomi, K., Kariyama, M., and Miyake, T. 2011. "Uniform Culture in Solid-state Fermentation with Fungi and its Efficient Enzyme Production." Journal of Bioscience and Bioengineering 111 (3): 300-5.

[6] Raimbault, M. 1998. "General and Microbiological Aspects of Solid State Fermentation." Electronic Journal of Biotechnology 1 (3): 1-13.

[7] Raimbault, M. 1997. "General Microbiological Aspects of Solid Substrate Fermentation." In: Proceedings of the International Course on Solid State Fermentation. Eds.: Raimbault, M., Soccol, C.R. and Chuzel, G. FMS97-6-10 October 1997, Curitiba, Brazil 1-20.

[8] Rajagopalan, S., and Modak, J. 1995. "Modelling of Heat and Mass Transfer for Solid State Fermentation Process in Tray Bioreactor." Bioprocess and Biosystems Engineering 13 (3): 161-9.

[9] Yang, S. T. 2007. "Bioprocessing for Value-added Products from Renewable Resources: New technologies and applications." Elsevier Science.

[10] Ariff, A. B., and Webb, C. 1996. "Influence of Different Fermenter Configurations and Modes of Operation on Glucoamylase Production by Aspergillus awamori." Asia Pasific Journal of Molecular Biology and Biotechnology 4: 183-95.

[11] Lie, S. 1973. "EBC [European Brewery Convention]-ninhydrin Method for Determination of Free $\alpha$-amino Nitrogen." Journal of Institute of Brewing, London 79: 37-41.

[12] Wang, R.-H. 1999. "Continuous Production of Generic Fermentation Feedstock from Whole Wheat Flour." Ph.D. Thesis. The University of Manchester Institute of Science and Technology, Manchester, United Kingdom. 

Opened and Closed System

[13] Bailey, M. J., Bieley, P., and Poutanen, K. 1992. "Interlaboratory Testing of Methods for Assay of Xylanase Activity." Journal of Biotechnology 23: 257-70.

[14] Miller, G. L. 1959. "Use of Dinitrosalicylic Acid reagent for Determination of Reducing Sugar." Analytical Chemistry 31 (3): 426-8.

[15] Stoilova, I., and Krastanov, A. 2008. "Overproduction of Laccase and Pectinase by Microbial Associations in Solid Substrate Fermentation." Applied Biochemical and Biotechnology 149: 45-51.

[16] Wang, R.-H., Shaarani, S. M., Godoy, L. C., Melikoglu, M., Vergara, C. S., Koutinas, A., and Webb, C. 2010. "Bioconversion of Rapeseed Meal for the Production of a Generic Microbial Feedstock." Enzyme and Microbial Technology 47: 77-83.

[17] Nahid, P., Vossoughi, M., Roostaazad, R., and Ahmadi, M. 2012. "Production of Glucoamylase by Aspergillus niger under Solid State Fermentation." IJE Transactions B: Applications 25 (1): 1-7.

[18] Mitra, P., Chakraborty, R., and Chandra, A. L. 1996. "Production of Proteolytic Enzymes by Solid-state Fermentation." Journal of Scientific and Industrial Research 55: 439-42.

[19] Babu, K. R. and Satyanarayana, T. 1996. "Production of Bacterial Enzymes by Solid-state Fermentation.” Journal of Scientific and Industrial Research 55: 464-7.

[20] Gupta, U., and Kar, R. 2008. "Optimization and Scale up of Cellulase Free Endo Xylanase Production by Solid State Fermentation on Corn Cob and by Immobilized Cells of a Thermotolerant Bacterial Isolate." Jordan Journal of Biological Science 1 (3): 129-34.

[21] Dhillon, G. S., Oberoi, H. S., Kaur, S., Bansal, S., and Brar, S. K. 2011. "Value-addition of Agricultural Wastes for Augmented Cellulose and Xylanase Production through Solid-state tray Fermentation Employing Mixed-culture of Fungi." Industrial Crops and Products 34: 1160-7.

[22] Favaro, L., Basaglia, M., and Casella, S. 2012. "Processing Wheat Bran into Ethanol using Mild Treatments and Highly Fermentative Yeasts." Biomass and Bioenergy 46: 605-17.

[23] Ellaiah, P., Adinarayana, K., Bhavani, Y., Padmaja, P., and Srinivasalu, B. 2002. "Optimization of Process Parameters for Glucoamylase Production under Solid State Fermentation by a Newly Isolated Aspergillus species." Process Biochemistry 38: 615-20.

[24] Hata, Y., Ishida, H., Kojima, Y., Ichikiwa, E., Kawato, A., Suginami, K., and Imayasu, S. 1997. "Comparison of Two Glucoamylases Produced by Aspergillus oryzae in Solid-state Culture (Koji) and in Submerged Culture." Journal of Fermentation Technology 84 (6): 532-7.

[25] Pandey, A. 1990. "Improvements in Solid State
Fermentation for Glucoamylase Production.” Biological Wastes 34: 11-9.

[26] Belmessikh, A., Boukhalfa, H., Mechakra-Maza, A., Gheribi-Aoulmi, Z., and Amrane, A. 2013. "Statistical Optimization of Culture Medium for Neutral Protease Production by Aspergillus oryzae: Comparative Study Between Solid and Submerged Fermentations on Tomato Pomace." Journal of the Taiwan Institute of Chemical Engineers 44: 377-85.

[27] Cai, J.-M., Wu, K., Zhang, J., and Pan, R.-R. 2006. "Production, Properties and Application of Xylanase from Aspergillus niger A3." Annals of the New York Academy of Sciences: Enzyme Engineering XIV 862: 214-8.

[28] Ridder, E. R., Nokes, S. E., and Knutson, B. L. 1999. "Optimization of Solid-state Fermentation Parameters for the Production of Xylanase by Trichoderma longibrachiatum on Wheat Bran in a Forced Aeration System." Transaction of the ASABE 42 (6): 1785-90.

[29] Gervais, P., and Molin, P. 2003. "The Role of Water the Water in Solid-state Fermentation." Biochemical Engineering Journal 13: 85-101.

[30] Archana, A., and Satyanarayana, T. 2003. "Purification and Characterization of a Cellulose-free Xylanase of a Moderate Thermophile Bacillus licheniformis A99." World Journal of Microbiology and Biotechnology 19: 53-7.

[31] Jain, A. 1995. "Production of Xylanase by Thermophilic Melanocarpus albomyces IIS-68." Process Biochemistry 30 (8): 705-9.

[32] Pandey, A., Soccol, C. R., Rodriguez-Leon, J. A., and Nigam, P. S. 2001. "Solid-state Fermentation in Biotechnology: Fundamentals and Application." First Edition. ASISTECH PUBLISHERS, INC. New Delhi.

[33] Pandey, A., Soccol, C. R., and Larroche, C. 2008. "Preface in Current Developments in Solid-state Fermentation." Eds.: Pandey, A., Soccol, C.R. and Larroche, C. Springer, Asiatech Publishers, Inc. New Delhi.

[34] Pandey, A., Soccol, C. R., and Larroche, C. 2010. "Current Developments in Solid-state Fermentation." Springer Science + Business Media, LLC.

[35] Corredor, D. Y. 2008. "Pretreatment and Enzymatic Hysdrolysis of Lignocellulosic Biomass." Ph.D. Thesis. Kansas State University, Manhattan, Kansas.

[36] Brijwani, K., and Vadlani, P. V. 2011a. "Cellulolytic Enzymes Production via Solid-state Fermentation: Effect of Pretreatment Methods on Physicochemical Characteristics of Substrate." SAGE-Hindawi Access to Research: Enzyme Research Vol. 2011, Article ID 860134, doi: 10.4061/2011/860134.

[37] Brijwani, K., Vadlani, P. V., Hohn, K. L., and Maier, D. E. 2011b. "Experimental and Theoretical Analysis of a 
Novel Deep-bed Solid-sate Bioreactor for Cellulolytic Enzymes Production." Biochemical Engineering Journal 58-59: 110-23.

[38] Amorea, A., and Faracoa, V. 2012. "Potential of Fungi as Category I: Consolidated BioProcessing Organisms for Cellulosic Ethanol Production." Renewable and Sustainable Energy Reviews 16: 3286-301.
[39] Viikari, I., Vehmaanpera, J., and Koivula, A. 2012. "Lignocellulosic Ethanol: From Science to Industry." Biomass Bioenergy 46: 13-24.

[40] Sudo, S., Kobayashi, S., Kaneko, A., Sato, K., and Oba, T. 1995. "Growth of Submerged Mycelia of Aspergillus kawachii in Solid-state Culture." Journal of Fermentation and Bioengineering 79 (3): 252-6. 